\title{
Rigid-body motion correction of the liver in image reconstruction for golden-angle stack-of-stars DCE MRI
}

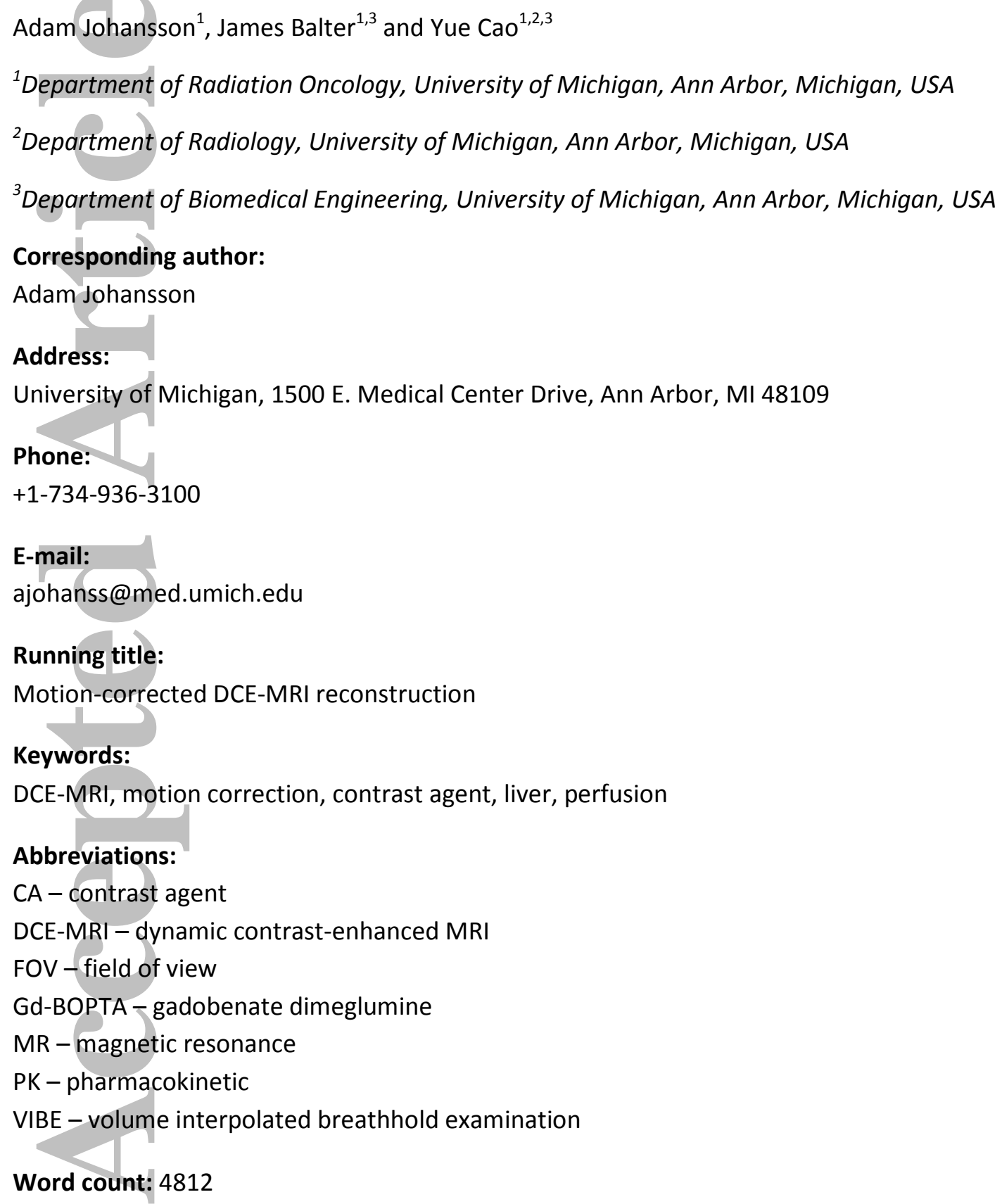

Table count: 2

This is the author manuscript accepted for publication and has undergone full peer review but has not been through the copyediting, typesetting, pagination and proofreading process, which may lead to differences between this version and the Version record. Please cite this article as doi:10.1002/ mrm.26782. 


\section{Abstract}

Purpose: Respiratory motion can affect pharmacokinetic perfusion parameters quantified from liver DCE-MRI. Image registration can be used to align dynamic images post-reconstruction. However, intraimage motion blur remains after alignment and can alter the shape of contrast-agent uptake curves. We introduce a method to correct for inter- and intra-image motion during image reconstruction.

Methods: 16 liver DCE-MRI examinations of 9 subjects were performed using a golden-angle stack-ofstars sequence. For each examination, an image time series with high temporal resolution but severe streak artifacts was reconstructed. Images were aligned using region-limited rigid image registration within a region of interest covering the liver. The transforms resulting from alignment were used to correct raw data for motion by modulating and rotating acquired lines in k-space. The corrected data was then reconstructed using view-sharing.

Results: Portal-venous input functions extracted from motion-corrected images had significantly greater peak signal enhancements (mean increase: $16 \%$, t-test, $\mathrm{p} \ll 0.001$ ) than those from images aligned using image registration after reconstruction. Also, portal-venous perfusion maps estimated from motioncorrected images showed fewer artifacts close to the edge of the liver.

Conclusion: Motion-corrected image reconstruction restores uptake curves distorted by motion. Motion correction also reduces motion artifacts in estimated perfusion parameter maps.

\section{Introduction}

The liver is supplied by blood from the hepatic artery and the portal vein. Arterial and portal-venous perfusion can provide different pieces of information about the state of the liver. Increases in the size of intrahepatic tumor subvolumes that demonstrate elevated arterial perfusion have been shown to predict tumor progression during radiotherapy (1) whereas portal-venous perfusion of normal liver parenchyma has been correlated with local and global liver function used to help assess the risk of radiation-induced liver disease (RILD) (2-5).

Liver perfusion can be quantified using dynamic contrast-enhanced magnetic resonance imaging (DCEMRI) (6). However, tissue motion during dynamic imaging, due to processes including breathing, peristalsis and heartbeat, can result in a given voxel not covering the same piece of tissue throughout an examination. This may lead to corruption of the contrast-agent (CA) uptake curves for imaged voxels, potentially confounding the analysis and mapping of perfusion parameters calculated from abdominal DCE images. In addition to spatial mismatch, motion can cause image blurring, signal loss, and other artifacts, such as streaks. The resulting uptake curves may then exhibit oscillations and spurious peaks that are not related to the CA dynamics, or they may have their amplitudes and shapes systematically altered by motion-induced partial volume effects.

To counteract the effects of motion, liver DCE-MRI has been acquired during repeated breath holding (5). However, some subjects may fail to hold their breath for a sufficient amount of time to sample the arterial and portal-venous input functions. Also, deep inhales taken in between breath holds introduce 
gaps in the CA uptake curves and such gaps may conceal important uptake-curve features. If a subject is instead asked to breathe freely during a DCE examination, large bulk motion as well as severe motion blur will often be observed. Consequently, it is necessary to compensate for motion as a part of image reconstruction or post-processing.

Region-limited rigid image registration has previously been used to align moving organs in images from DCE-MRI (7). However, motion-induced image blur still remains after image alignment if temporal resolution is insufficient. A high temporal resolution is also needed to faithfully capture the CA uptake dynamics. Therefore, extensive efforts have gone into developing imaging methods that can acquire images faster without sacrificing spatial resolution. Many of these imaging methods use stack-of-stars or stack-of-spirals sampling schemes combined with view sharing, parallel imaging or compressed sensing to push the boundary of spatiotemporal resolution (8-11) or to produce more benign artefacts, such as blurring, rather than streaks and ghosts (12-15). Yet, even at the high temporal resolutions seen to date, some intra-image motion blur can persist. To compensate for such motion-induced artifacts within an image, respiratory self-gated $\mathrm{k}$-space data has been corrected for translation by minimizing the regional image entropy $(16,17)$.

In this work we introduce a method to correct for inter- and intra-image motion as a part of the imagereconstruction process. We first propose a motion signal generated from rigidly aligning images reconstructed from severely undersampled data. In this context, undersampling supports image creation with a temporal resolution sufficient to resolve the breathing cycle. Secondly, the motion signal, consisting of a time series of rigid transforms (translation and rotation), is used to correct k-space data for rigid motion. Finally, the corrected data is reconstructed into DCE-MRI time series using temporal view sharing. The method is evaluated by comparing portal-venous input functions and portal-venous perfusion maps derived from images reconstructed from motion-corrected k-space data to those derived from images aligned using image registration after reconstruction.

\section{Theory}

In MRI acquisition, the raw samples collected in k-space by an imaging sequence can be interpreted as samples of the Fourier transform, $s(\mathbf{k})=\mathcal{F} S(\mathbf{k})$, of a sought image, $S(\mathbf{r})$, where $\mathbf{k}=\left(k_{x}, k_{y}, k_{z}\right)^{\mathrm{T}}$ is a position in $\mathrm{k}$-space and $\mathbf{r}=(\mathrm{x}, \mathrm{y}, \mathrm{z})^{\mathrm{T}}$ a position in image space. If motion occurs during acquisition, the image $S(\mathbf{r}, t)$ and its Fourier transform $s(\mathbf{k}, t)$ will depend on time. Affine motion can be thought of as a dynamic spatial transformation of a motionless image $\tilde{S}(\mathbf{r})$ such that

$$
\tilde{S}(\mathbf{r})=S(A(t) \mathbf{r}+\mathbf{b}(t), t)
$$

where $A(t)$ is a time-dependent $3 \times 3$ transformation matrix and $\mathbf{b}(t)$ is a time-dependent translation vector. For the case of rigid-body motion, $A(t)$ is a rotation matrix. In k-space, the effect of the transformation in (1) is a combination of a linear transformation of $\mathbf{k}$ and a signal modulation. Dropping the $t$ for notational simplicity, the Fourier transform $\tilde{s}(\mathbf{k})=\mathcal{F} \tilde{S}(\mathbf{k})$ can be written (16-18) as

$$
\mathcal{F}(\mathbf{r} \mapsto S(A \mathbf{r}+\mathbf{b}))(\mathbf{k})=\exp \left(2 \pi i \mathbf{b} \cdot A^{-\mathrm{T}} \mathbf{k}\right) \times \frac{1}{|\operatorname{det} A|} S\left(A^{-\mathrm{T}} \mathbf{k}\right) .
$$


Therefore, a motion-corrupted sample $s_{i}=s\left(\mathbf{k}_{i}\right)$ collected at position $\mathbf{k}_{i}$ can be transformed into a motion-corrected sample $\tilde{s}_{i}$ with apparent sample position $\tilde{\mathbf{k}}_{i}$ using the following relations.

$$
\begin{aligned}
& \tilde{s}_{i}=\exp \left(2 \pi i \mathbf{b} \cdot \mathbf{k}_{i}\right) s_{i} \\
& \tilde{\mathbf{k}}_{i}=A^{\mathrm{T}} \mathbf{k}_{i}
\end{aligned}
$$

Equations ( 3 ) and (4) provide means to correct motion-corrupted k-space data before reconstruction if $A(t)$ and $\mathbf{b}(t)$ are known.

\section{Methods}

A framework to perform motion-corrected reconstruction of a DCE-MRI time series from k-space data acquired by a gradient-echo stack-of-stars sequence with golden-angle sampling is illustrated by the flowchart in Fig. 1. The framework consists of five major processing steps: 1) pre-processing of raw kspace data, 2) reconstruction of dynamic images with high temporal resolution, 3 ) creation of a motion signal $(A(t), \mathbf{b}(t))$ through image registration of the high temporal resolution images, 4) motion correction of k-space data, and 5) final reconstruction of the 3D time series with a tornado filter (19). The different components of the processing pipeline are described in the following subsections.

Imaging

A prototype gradient-echo stack-of-stars sequence with golden-angle sampling (Radial VIBE, Siemens Healthineers, Erlangen, Germany) was used for scanning $(20,21)$. This sequence was provided as a workin-progress package by the vendor for evaluation. As the sampling pattern illustrated in Fig. 2 shows, radial readout lines are acquired in the $x-y$ plane with a golden angle scheme while Cartesian phase encoding is performed in the $k_{z}$ direction. Also, readout lines at each angle are acquired for all $k_{z}$ values before progressing to the next angle, as illustrated by the dark gray rectangle in Fig. 2 . The lines in this rectangle are collectively referred to as a spoke. The sampling pattern was oriented such that radial sampling was parallel to the transverse plane and Cartesian sampling was along the superior-inferior direction in k-space.

\section{Reconstruction with high temporal resolution}

Raw data was collected from the scanner and partitioned into successive groups of 5 spokes. A threedimensional volume was reconstructed using gridding $(22,23)$ for each group of spokes. Prior to gridding reconstruction, a number of adjustments were made to the raw data. Small gradient delays have little effect on Cartesian imaging but can lead to artifacts for radial sampling schemes if not corrected for (24). Therefore the gradient delay was estimated by fitting a straight line to the phase difference of radial readout lines acquired in almost opposite direction. The slope of this line is proportional to the size of the gradient delay. As an approximation, this delay was assumed to be independent of the readout direction. Readout lines were corrected by shifting them along the readout direction such that the slope of the phase difference became zero. Missing k-space lines were synthesized using an iterative partial-Fourier reconstruction method (25). The noise covariance for the receiver coils was estimated using samples collected at the edge of k-space. The resulting covariance matrix was then used to 
perform a whitening transform of the collected data. The result of this noise decorrelation was to produce a set of synthetic coil signals with independent noise (26). These synthetic coil signals were then used in place of the original signals. A seven-grid-sample-wide cubic Kaiser-Bessel kernel was used for gridding. The k-space grid was oversampled by 37.5 percent compared to the inverse of the nominal field of view (22). Coil images were combined for optimal signal-to-noise ratio using estimated coil sensitivity profiles (27). These profiles were estimated by dividing coil images reconstructed from 500 spokes at the latter half of the examination with the root-sum-of-squares image. Each reconstructed volume was assigned a timestamp equal to the timestamp of the acquisition line passing through the center of $k$-space for the third spoke in its five spoke group.

\section{Image registration}

The reconstructed time series was aligned using region-limited rigid registration with a mutual information metric defining the optimization cost function for alignment. Because of the severe aliasing caused by using only five spokes, simple registration to one reference $3 \mathrm{~d}$ image was not sufficient. Instead each image was registered to twenty other randomly selected images within the high-temporalresolution time series to form an overdetermined system of transform equations. This system was then solved in the $L_{1 / 2}$ sense using iteratively reweighted least squares (IRLS) (28) to reject outlier transforms caused by bad alignments (29). The registration metric was only evaluated within an ROI drawn to cover the liver as it appeared in a reference volume previously selected by a physician to use for radiation therapy treatment planning guidance due to its lesion contrast. The registration produced a set of rigid transforms describing the relative position and orientation of the liver in each volume with respect to the reference volume.

\section{Raw data motion correction}

The transform time series produced by registration was interpolated using the matrix logarithm (30) to form one rigid transform for each spoke. The matrix logarithm was used to produce a smooth rigid body motion in $3 \mathrm{~d}$ space. The acquired k-space data was then corrected for motion using equations ( 3 ) and (4). Data from each coil was corrected separately and no correction for the shift of the coil sensitivity profiles with respect to anatomy was applied.

\section{Reconstruction with tornado filter}

The radial sampling schemes used leads to a denser sampling of the center of k-space than the outer parts. After motion correction the raw data was reconstructed into a time series using a tornado filter (19) that included temporally neighboring samples to ensure that the sampling density met the Nyquist criterion for a majority of k-space. The tornado filter therefore compensates for the difference in sampling densities between the center and outer parts of k-space by sharing samples from neighboring temporal views thereby reducing aliasing at the cost of reduced temporal resolution for higher spatial frequencies. The number of spokes needed for k-space to be fully sampled was determined by the single spoke density compensation weights, $v(\mathbf{k})$, which equals the k-space volume of a semicylindrical shell with central radius $|\mathbf{k}|$ and with height and shell width equal to the sample spacing in the longitudinal and radial directions respectively. To reduce ringing, the effective resolution of the image was reduced 
slightly below the resolution of the nominal voxel size by reducing the weights with a Gaussian k-space window

$$
G(\mathbf{k})=e^{-2 \pi \sigma^{2}\|\mathbf{k}\|^{2}}
$$

with $\sigma=1 / 3$ voxels, such that a new density compensation $w_{0}(\mathbf{k})=v(\mathbf{k}) G(\mathbf{k})$ is formed. With respect to time the tornado filter was not a rect-function as in (19) but had the shape of a truncated Gaussian

$$
T(\mathbf{k}, j)=\frac{w_{0}}{w} \exp \left(-\pi\left(\frac{j-j_{0}}{w}\right)^{2}\right) \operatorname{rect}\left(\frac{j-j_{0}}{w_{\max }}\right)
$$

where $j$ is the spoke index and $j_{0}$ the index of the centermost spoke of the current volume being reconstructed. The width $w$ of the Gaussian in Eq. 6 was given by

$$
w(\mathbf{k})=\left(\frac{1}{w_{0}^{2}+w_{\min }^{2}}+\frac{1}{w_{\max }^{2}}\right)^{-1 / 2}
$$

such that the width was never below $w_{\min }=21$ spokes or above $w_{\max }=144$ spokes. Filter weights were calculated based on the golden angle sampling pattern and no corrections of the weights were applied for the shift of apparent sample locations caused by motion correction. Aside from correcting the data for motion and replacing the 5-spoke sliding window filter with a tornado filter, other parts of the reconstruction chain, such as partial Fourier reconstruction and optimal coil combining, were identical to those used for reconstruction of images with high temporal resolution.

\section{Evaluation}

Under institutional review board approval, 16 DCE-MRI examinations with raw k-space data from 9 patients were included in this study (women, 2; men, 7; age at examination, 48-76; number of examinations per patient, 1-3). As the study investigates the possibility of individualized adaptive radiotherapy for hepatocellular carcinoma, all subjects had tumors in the liver. Subjects were scanned for about 5 min on a 3 T MRI scanner (Magnetom Skyra, Siemens Healthineers, Erlangen, Germany) while breathing freely. After $30 \mathrm{~s}$ of scanning $20 \mathrm{ml}(0.5 \mathrm{M})$ of a Gd-BOPTA-based contrast agent (MultiHance, Bracco Diagnostics Inc., Monroe, NJ) was administered intravenously. Sequence parameters are shown in Table 1 . They were allowed to vary slightly among patients to ensure liver coverage and avoid system-reported patient-heating issues.

Images reconstructed with our motion-corrected reconstruction algorithm were compared to images reconstructed without motion correction but aligned in the image domain after reconstruction using region-limited rigid-body image registration (29). Two sets of images without motion correction were used for this purpose. The first set was reconstructed identically to the motion corrected images but with $A(t)=I$ and $b(t)=(0,0,0)^{\mathrm{T}}$, i.e. assuming no subject motion. The second set of images was reconstructed by vendor software on the scanner employing KWIC (12) without motion correction.

For each set of images, time series portal-venous input functions were extracted and portal-venous perfusion maps estimated using a dual-input single-compartment perfusion model (6). The portalvenous perfusion maps were compared across the reconstruction algorithms qualitatively. Also, the 
maximum signal enhancement was determined for each extracted portal-venous input function and used as a quantitative evaluation metric. This metric was chosen because the portal vein is a small structure that appears brighter than surrounding liver tissue before contrast administration and darker than surrounding tissue after administration. Therefore motion-induced blurring will reduced the signal enhancement from pre- to post-contrast images. The arterial input function used for perfusion estimation was measured in the aorta, which moves less than the liver during breathing, and thus was extracted from images that were not motion corrected or registered.

\section{Reconstruction of motion states}

Images showing the different states of the breathing cycle can be used to characterize the spatial transformation that organs undergo. In order to reconstruct images representative of the average configuration and position of the liver at various points in the breathing cycle, the range of values for the translational displacement of the liver in the superior-inferior direction was divided into five intervals of equal length. Motion corrected raw data was then sorted according to what interval it belonged to and one motion state was reconstructed from all spokes in each interval. The inhale motion states were aligned to the exhale states with deformable registration using NiftyReg(31). The resulting deformation vector fields then show the residual deformation after rigid alignment. The median as well as the 95 percentile of the magnitude of the residual displacement was determined for each patient.

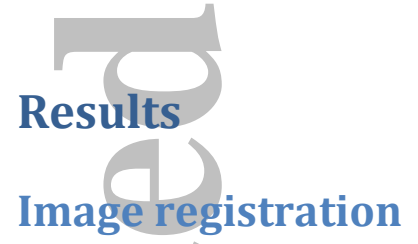

Images reconstructed from five spokes showed streak artifacts due to undersampling. However, the image quality was still sufficient for rigid alignment as seen in Fig. 3.

An example of a motion signal measured from a subject is shown in Fig. 4. The largest translational motion is seen along the superior-inferior direction and the largest rotational motions occur about the anterior-posterior and left-right axes. The mean range of motion measured from all studies is listed in Table 2.

\section{Reconstruction using the tornado filter}

The effect of k-space motion correction on images reconstructed with the tornado filter is seen in Fig. $5 a-f$. The same images reconstructed by the vendor software on the scanner are also shown (Fig. 5g-i). The k-space motion corrected images exhibit less motion blur, particularly at the dome and inferior tip of the liver, and for small structures, e.g., the portal vein (Fig. 5b). The images reconstructed on the scanner show similar motion artifacts to those from the uncorrected tornado reconstruction.

\section{Impact on measured contrast dynamics and perfusion maps}

Fig. 6 shows the time-intensity curves of an ROI drawn to determine the portal-venous input function (PVIF) for two subjects. The signal enhancement compared to the baseline is larger for the motioncorrected images than for the uncorrected ones due to reduced motion-induced partial-volume effects. The maximum signal enhancement from the pre-contrast baseline to the peak of the portal-venous 
input function was determined for each patient with and without motion correction. For all examinations the maximum signal enhancement was higher for the motion corrected input function (mean increase: 16\%, range of increase: [5\%, 35\%], 95\% confidence interval of the mean increase: [12\%, $20 \%]$ ). The mean increase of the signal enhancement was significantly higher (significance level: $5 \%$ ) for motion corrected images (Student's t-test, $p \ll 0.001$ ). Similar results were found when comparing motion-corrected to vendor-reconstructed images (mean increase: $20 \%, p \ll 0.001$ ). No significant difference was found between the mean maximum signal enhancements for the vendor-reconstructed images and the tornado-reconstructed images without motion correction (mean difference: $-2 \%, p=$ $0.3)$.

Transverse perfusion parameter maps for a subject are shown in Fig. 7. The motion-corrected PVIF and liver uptake curves produced a portal-venous perfusion parameter map (Fig. 7f) that differed markedly from the parameter maps produced by the uncorrected images (Fig. 7d-e). A prominent artifact in Fig. $7 e$ is the low perfusion in the posterior part of the liver (bottom of image). Here, motion of surrounding tissue into and out of the liver ROI causes an erroneous estimated reduced venous perfusion. Similar perfusion bias artifacts were observed for several of the subjects included in this study.

\section{Motion states}

Three of the five reconstructed motion states for one subject are shown in Fig. 8. These motion states show sharp liver edges and internal structures. They also show that residual motion remains for the inferior tip of the liver after rigid-body motion correction. Because translation and rotation have been corrected for, the remaining modes of motion are the deformations that the liver undergoes during the breathing cycle, such as bending or compression. These cannot be corrected for using rigid-body registration, but require deformable image registration instead. The median residual displacement after rigid alignment as determined using deformable alignment was found to vary between 2.2 and $6.9 \mathrm{~mm}$ with a mean of $3.0 \mathrm{~mm}$ among patients. The 95th percentile of the residual displacement varied between $4.6 \mathrm{~mm}$ and $15.7 \mathrm{~mm}$. For two examinations the 95th percentile of the residual displacement was larger than $10 \mathrm{~mm}$. For 10 examinations the 95th percentile was larger than $6 \mathrm{~mm}$ or two voxels.

\section{Discussion}

Respiratory motion introduces organ displacements as well as streaking and blurring into images reconstructed from DCE-MRI examinations. Unless corrected for, these effects can distort CA uptake curves and cause error in estimated perfusion parameter maps of the liver. Previous studies have shown that partitioning acquired spokes into smaller sets before reconstruction can help to reduce image blurring. The reduction in set sizes can be achieved by a higher temporal resolution or by introducing an extra motion-state dimension to complement the spatial and temporal dimensions. In both cases the reduction in set sizes must be accompanied by more advanced reconstruction methods such as throughtime GRAPPA (10) or compressed sensing (9) to counteract the more severe streak artifacts that would otherwise result. Rather than partitioning acquired data into smaller sets, the presented study builds upon earlier work (16) to perform motion correction in k-space before reconstruction. Motion-corrected DCE-MRI k-space data can then be reconstructed into time series with arbitrary temporal resolution (14) 
or into dedicated arterial and portal-venous phase images without motion artifacts. In the radiation therapy clinic this versatility could produce an image time-series for perfusion estimation as well as a smaller set of images with less aliasing and noise for tumor delineation.

The robust image registration algorithm used to align the high-temporal-resolution images in this study was able to produce a rigid-body motion signal that reflected the semi-periodic pattern of respiratory motion despite the changing image contrast introduced by the contrast agent dynamics. Because the motion signal was derived from registration of each image in the whole time series it does not enforce periodicity in the breathing pattern. By applying the rigid-body motion correction to the acquired kspace data, time-series images with reduced motion blur were reconstructed. After motion correction, the portal-venous input functions demonstrated larger maximum signal enhancement for all of the DCEMRI examinations in this study. This can be understood by considering that the portal vein is darker than surrounding liver tissue prior to CA administration. After administration it appears brighter due to its higher CA concentration. Motion-induced partial volume effects will therefore reduce the dynamic range of the signal enhancement in the portal vein. By correcting for motion, the higher signal enhancement of the portal vein is recovered. This ability to recover contrast lost due to blurring is an advantage over other free-breathing imaging techniques that attempt to reduce the severity of motioninduced artifacts by promoting motion blur over streaks and ghosting through temporal regularization (8) or view sharing (13) without motion correction. Since the motion of the portal vein is similar to that of the liver it is reasonable to assume that the signal enhancements of smaller structures, such as blood vessels and lesions, are also smeared due to breathing, leading to a potential loss of lesion contrast.

Only respiratory and slower motions as they affect the liver were characterized and corrected for in this study. Cardiac motion cannot currently be resolved by the presented technique and was therefore not corrected for. No assumption about the pattern of motion, such as periodicity, was assumed and the method was therefore also able to characterize and correct for slow drifts in liver position throughout the 5 min examination. Including other modes of motion such as cardiac and gastrointestinal in our motion model is a focus of future research.

Parameter maps estimated from motion-corrected images showed fewer motion artifacts than those from non-corrected images. Visually, the anatomic structure of the liver was more consistently represented on motion-corrected images. This was particularly true for the dome and lower tip of the liver that move in and out of regions covered by other organs with minimal contrast uptake and thus lower intensity.

Because the temporal width of the tornado filter depends on the distance from the center of k-space, the temporal resolution of different structures in the images will depend on their size and texture. At the center of k-space the temporal resolution will be about $3.2 \mathrm{~s}$ whereas the edge of $\mathrm{k}$-space only will have a temporal resolution of about $22 \mathrm{~s}$. Larger structure, like the aorta and homogenous pieces of liver will have a higher temporal resolution, whereas thinner arteries within the liver will have lower temporal resolution. This could affect uptake curves (10), especially for thin arteries but should have little effect for liver parenchyma where the uptake dynamics have already been filtered to a lower temporal frequency content, or for the aorta which is large enough to accurately reflect the more rapid 
changes in CA concentration. For the portal vein the effective temporal resolution is about $7.7 \mathrm{~s}$. This is lower than what would be needed to represent the AIF from the aorta, but as the portal-venous input function contains less rapid dynamics, the initial rise and the slowly decaying plateau can be accurately represented at this resolution.

The coil sensitivity maps used to combine coil images were estimated using a rather simple method. Because these profiles were only used to combine coil images through voxel-wise weighting the only effect from errors in sensitivity is a small potential reduction in SNR. However if parallel-imaging techniques were to be included in the reconstruction method, more accurate sensitivity maps would be needed (32).

The rigid-body motion correction used in this work cannot compensate for deformations of the liver (such as bending). Nonetheless, it does correct for the majority of the displacement experienced by most parts of the liver and therefore improves the sharpness of reconstructed images. For most patients, the residual deformation as determined by deformable registration between inhale and exhale states was in the order of a voxel. However, for 2 examinations of 16 , at least $5 \%$ of the liver showed residual motion larger than $10 \mathrm{~mm}$. Residual nonrigid-body motion of this larger magnitude could influence perfusion quantification in the affected area. If needed, the motion corrected motion state images presented in this work could be used as a starting point for deformable registration and iterative model-based reconstruction (33). These motion state images exhibit less motion blur for inhale phases than their non-motion-corrected counterparts and could therefore provide sharper image details to help guide deformable image registration. Ongoing studies are investigating applications of such deformable motion correction. We have found that deformable alignment would be needed for about $13 \%$ and $60 \%$ of the examinations in this work in order to bring the 95th percentile of the residual displacement below $10 \mathrm{~mm}$ and $6 \mathrm{~mm}$, respectively, for all examinations.

\section{Conclusions}

A framework for DCE-MRI time series reconstruction with rigid-body motion correction of the liver position and orientation was presented. A robust image-space registration procedure was used to produce a rigid-body motion signal that was insensitive to the CA uptake dynamics. Employed in the presented motion-corrected reconstruction algorithm for acquired k-space data, alignment helped reduce motion blur and large displacements in DCE-MRI time series of the liver. Consequently, partial volume effects were reduced and higher maximum signal enhancements were recovered for the portalvenous input functions for all DCE-MRI examinations in the study. In addition, motion correction reduced artifacts in estimated portal-venous perfusion maps, in particular for regions close to the edge of the liver. For $13 \%$ of subjects, at least $5 \%$ of the liver showed residual deformation in excess of $10 \mathrm{~mm}$ after motion correction. For these cases, the derived motion signal can be used to reconstruct sharpened breathing state images in preparation for image reconstruction with deformable motion correction. 


\section{Acknowledgements}

This work is supported in part by NIH/NCI RO1 CA132834 and PO1 CA059827. The prototype Radial VIBE sequence was provided by Siemens Healthineers under a research agreement.

\section{Disclosure}

No disclosures to report.

\section{References}

1. Wang H, Farjam R, Feng M, Hussain H, Ten Haken RK, Lawrence TS, Cao Y. Arterial Perfusion Imaging-Defined Subvolume of Intrahepatic Cancer. Int J Radiat Oncol Biol Phys. 2014;89(1):16774.

2. Cao Y, Platt JF, Francis IR, Balter JM, Pan C, Normolle D, Ben-Josef E, Haken RK Ten, Lawrence TS. The prediction of radiation-induced liver dysfunction using a local dose and regional venous perfusion model. Med Phys. 2007;34(2):604-12.

3. Cao Y, Pan C, Balter JM, Platt JF, Francis IR, Knol J a, Normolle D, Ben-Josef E, Ten Haken RK, Lawrence TS. Liver function after irradiation based on computed tomographic portal vein perfusion imaging. Int J Radiat Oncol Biol Phys. 2008;70(1):154-60.

4. CaoY. The Promise of Dynamic Contrast-Enhanced Imaging in Radiation Therapy. Semin Radiat Oncol. 2011;21(2):147-56.

5. Cao Y, Wang H, Johnson TD, Pan C, Hussain H, Balter JM, Normolle D, Ben-Josef E, Ten Haken RK, Lawrence TS, Feng M. Prediction of liver function by using magnetic resonance-based portal venous perfusion imaging. Int J Radiat Oncol Biol Phys. 2013;85(1):258-63.

6. Wang H, Cao Y. GPU-accelerated voxelwise hepatic perfusion quantification. Phys Med Biol. 2012;57(17):5601-16.

7. Zuo CS, Jiang A, Buff BL, Mahon TG, Wong TZ. Automatic motion correction for breast MR imaging. Radiology. 1996;198(3):903-6.

8. Feng L, Grimm R, Tobias Block K, Chandarana H, Kim S, Xu J, Axel L, Sodickson DK, Otazo R. Golden-angle radial sparse parallel MRI: Combination of compressed sensing, parallel imaging, and golden-angle radial sampling for fast and flexible dynamic volumetric MRI. Magn Reson Med. 2014;72:707-17.

9. Feng L, Axel L, Chandarana H, Block KT, Sodickson DK, Otazo R. XD-GRASP: Golden-angle radial MRI with reconstruction of extra motion-state dimensions using compressed sensing. Magn Reson Med. 2015;75(2):775-88.

10. Chen Y, Lee GR, Wright KL, Badve C, Nakamoto D, Yu A, Schluchter MD, Griswold MA, Seiberlich $\mathrm{N}$, Gulani V. Free-breathing liver perfusion imaging using 3-dimensional through-time spiral generalized autocalibrating partially parallel acquisition acceleration. Invest Radiol. 2015;50(6):367-75.

11. Benkert T, Feng L, Sodickson DK, Chandarana H, Block KT. Free-Breathing Volumetric Fat/Water Separation by Combining Radial Sampling, Compressed Sensing, and Parallel Imaging. Magn 
Reson Med. 2016;0:1-12.

2. Song HK, Dougherty L. k-Space weighted image contrast (KWIC) for contrast manipulation in projection reconstruction MRI. Magn Reson Med. 2000;44(6):825-32.

3. Kim KW, Lee JM, Jeon YS, Kang SE, Baek JH, Han JK, Choi BI, Bang YJ, Kiefer B, Block KT, Ji H, Bauer $\mathrm{S}$, Kim C. Free-breathing dynamic contrast-enhanced MRI of the abdomen and chest using a radial gradient echo sequence with K-space weighted image contrast (KWIC). Eur Radiol. 2013;23:1352-60.

4. Riffel P, Zoellner FG, Budjan J, Grimm R, Block TK, Schoenberg SO, Hausmann D. “One-Stop Shop." Invest Radiol. 2016;51(11):714-9.

5. Parikh N, Ream JM, Zhang HC, Block KT, Chandarana H, Rosenkrantz AB. Performance of simultaneous high temporal resolution quantitative perfusion imaging of bladder tumors and conventional multi-phase urography using a novel free-breathing continuously acquired radial compressed-sensing MRI sequence. Magn Reson Imaging. 2016;34(5):694-8.

6. Lin W, Guo J, Rosen MA, Hee KS. Respiratory motion-compensated radial dynamic contrastenhanced (DCE)-MRI of chest and abdominal lesions. Magn Reson Med. 2008;60(5):1135-46.

7. Atkinson D, Hill DL, Stoyle PN, Summers PE, Keevil SF. Automatic correction of motion artifacts in magnetic resonance images using an entropy focus criterion. IEEE Trans Med Imaging. 1997;16(6):903-10.

8. Shechter G. Respiratory motion of the heart: Implications for magnetic resonance coronary angiography. Med Phys. 2004;31(6):1647.

9. Barger A V, Block WF, Toropov Y, Grist TM, Mistretta CA. Time-Resolved Contrast-Enhanced Imaging With Isotropic Resolution and Broad Coverage Using an Undersampled 3D Projection Trajectory. Magn Reson Med. 2002;48:297-305.

0. Block KT, Chandarana H, Milla S, Bruno M, Mulholland T, Fatterpekar G, Hagiwara M, Grimm R, Geppert C, Kiefer B, Sodickson DK. Towards routine clinical use of radial stack-of-stars 3D gradient-echo sequences for reducing motion sensitivity. J Korean Soc Magn Reson Med. 2014;18(2):87-106.

1. Chandarana H, Block KT, Winfeld MJ, Lala S V., Mazori D, Giuffrida E, Babb JS, Milla SS. Freebreathing contrast-enhanced T1-weighted gradient-echo imaging with radial k-space sampling for paediatric abdominopelvic MRI. Eur Radiol. 2014;24:320-6.

2. Beatty PJ, Nishimura DG, Pauly JM. Rapid gridding reconstruction with a minimal oversampling ratio. IEEE Trans Med Imag. 2005 Jun;24(6):799-808.

3. Jackson JI, Meyer CH, Nishimura DG, Macovski A. Selection of a Convolution Function for Fourier Inversion Using Gridding. IEEE Trans Med Imag. 1991;10(3):473-8.

4. Block KT, Uecker M. Simple method for adaptive gradient-delay compensation in radial MRI. Proc Int Soc Magn Reson Imaging. 2011;19:2816.

5. Cuppen J, van Est A. Reducing MR imaging time by one-sided reconstruction. Magn Reson Imaging. 1987;5(6):526-7.

6. Martini N, Santarelli MF, Giovannetti G, Milanesi M, De Marchi D, Positano V, Landini L. Noise correlations and SNR in phased-array MRS. NMR Biomed. 2010;23(1):66-73. 
27. Roemer PB, Edelstein WA, Hayes CE, Souza SP, Mueller OM. The NMR phased array. Magn Reson Med. 1990;16(2):192-225.

28. Gentle JE. Matrix Algebra Theory, Computations, and Applications in Statistics. Springer. 2007.

29. Johansson A, Balter J, Feng M, Cao Y. An overdetermined system of transform equations in support of robust DCE-MRI registration with outlier rejection. Tomography. 2016;2(3):188-96.

30. Alexa M. Linear combination of transformations. ACM Trans Graph. 2002;21(3):380-7.

31. Modat M, Ridgway GR, Taylor ZA, Lehmann M, Barnes J, Hawkes DJ, Fox NC, Ourselin S. Fast freeform deformation using graphics processing units. Comput Meth Prog Bio. 2010;98(3):278-84.

32. Fair MJ, Gatehouse PD, Drivas P, Firmin DN. Improved dynamic parallel imaging coil calibration method robust to respiratory motion with application to first-pass contrast-enhanced myocardial perfusion imaging. Magn Reson Med. 2016;75(6):2315-23.

33. Filipovic M, Vuissoz P -a., Codreanu a., Claudon M, Felblinger J. Motion compensated generalized reconstruction for free-breathing dynamic contrast-enhanced MRI. Magn Reson Med. 2011;65(3):812-22.

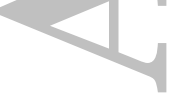

\section{Figure câptions}

FIG. 1. Motion-corrected image reconstruction pipeline. The raw k-space data from the MRI scanner (upper left corner) is reconstructed into a motion-corrected DCE-MRI time series (lower right corner).

FIG. 2. Golden-angle stack-of-stars k-space sampling pattern used by the Radial VIBE sequence. Straight black radial lines represent readout lines in k-space. The top light gray disc touches all lines belonging to one particular $\mathrm{k}_{\mathrm{z}}$ phase encoding index and the vertical dark gray rectangle touches all lines with a particular spoke index.

FIG. 3. Example of images reconstructed from five spokes before $(a, b)$ and after $(c, d)$ registration. The reference volume on which the liver $\mathrm{ROI}$ used for registration was drawn $(e, f)$ is also shown. This reference image was reconstructed without motion correction from a large number of spokes. The dashed white line shows the position of the liver in the reference volume.

FIG. 4. Rigid-body motion signal components with respect to time. The first three components ( $x, y$ and $z$ - left, posterior and superior in $\mathrm{mm}$ ) describe the 3D translation of the center of the liver. The last three components $\left(\phi_{x}, \phi_{y}\right.$ and $\phi_{z}$ in degrees) are the elements of an axis-angle representation of the rotation in degrees.

FIG. 5. Images reconstructed using the tornado filter with k-space motion correction (a, b, c) and without motion correction (d, e, f).Vendor-reconstructed images are shown in (g) through (i). The left column (a, $d, g)$ shows pre-contrast and the middle column $(b, e, h)$ post-contrast images in the coronal plane for one subject. The arrow in (b) points to the portal vein which appears as a bright spot. The right column $(c, f, i)$ shows how motion artifacts appear in the transverse plane for another subject. Streak and 
motion artifacts in (i) obscure the portal vein. In (f) streak artifacts are reduced by the tornado filter and in (c) motion correction further enhances the contrast between the portal vein and surrounding tissue.

FIG. 6. Portal-venous input functions from two different patients are shown in (a) and (b). For each patient, input functions derived from motion-corrected (MC) and non-motion-corrected (NMC) images reconstructed with the tornado filter are shown as well as NMC images reconstructed by vendor software on the scanner. NMC images were aligned using rigid image registration before extraction of the input functions. Input function from MC images exhibit larger maximum signal enhancements and are smoother compared to their NMC counterparts. The temporal distance between images is about $1 \mathrm{~s}$ for the tornado images and about $3 \mathrm{~s}$ for the vendor images. However, the effective temporal resolution for the tornado images is about $7.7 \mathrm{~s}$ in the portal vein due to the width of the tornado filter.

FIG. 7. Transverse arterial $(a-c)$ and portal-venous ( $d-f)$ perfusion maps calculated from DCE-MRI time series reconstructed by vendor software on the scanner without (first column), by a tornado filter without motion correction (second column) and by a tornado filter with motion correction (third column) for a subject. The portal-venous perfusion map in (e) exhibits severe artifacts caused by motion. Perfusion is given in $\mathrm{ml} /(100 \mathrm{ml} \cdot \mathrm{min})$.

FIG. 8. Three ((a) first, (b) third and (c) fifth) of five reconstructed motion states from an example subject as well as the superior-inferior motion that was used for sorting k-space data into states (d).The dotted white ROI indicates the liver contour in the reference volume. The white arrow in (a) points to the location of a tumor. Because the motion states were corrected for rigid-body motion the remaining differences are the result of deformations, such as bending.

\section{Tables}

Table 1. Sequence parameters used for DCE-MRI imaging with Radial VIBE.

\begin{tabular}{ll}
\hline \hline Sequence Parameter & Value \\
\hline Echo time & $1.16 \mathrm{~ms}-1.22 \mathrm{~ms}$ \\
Repetition time & $2.72 \mathrm{~ms}-4.00 \mathrm{~ms}$ \\
Flip angle & $11^{\circ}-14^{\circ}$ \\
Image matrix size & $192 \times 192$ \\
Number of slices & 64 \\
Number of phase encoding steps & 46 \\
Number of radial spokes & 2000 \\
Voxel size, in-plane (transversal) & $2 \mathrm{~mm}-2.3 \mathrm{~mm}$ \\
Slice thickness & $3 \mathrm{~mm}-4 \mathrm{~mm}$ \\
\hline \hline
\end{tabular}

Table 2. The population means and standard deviations of the within-examination motion ranges for different modes of motion.

\begin{tabular}{ll}
\hline \hline Type of motion & Mean range (standard deviation) of motion \\
\hline Translation, left-right, $x$ & $3.6(1.1) \mathrm{mm}$
\end{tabular}


Translation, posterior-anterior, $y$

Translation, superior-inferior, $z$

Rotation, sagittal plane, $\phi_{x}$

Rotation, coronal plane, $\phi_{y}$

Rotation, transverse plane, $\phi_{z}$
$9.1(4.2) \mathrm{mm}$

19.9(9.1) $\mathrm{mm}$

$4.2(1.8)^{\circ}$

$4.0(2.0)^{\circ}$

$3.3(1.3)^{\circ}$
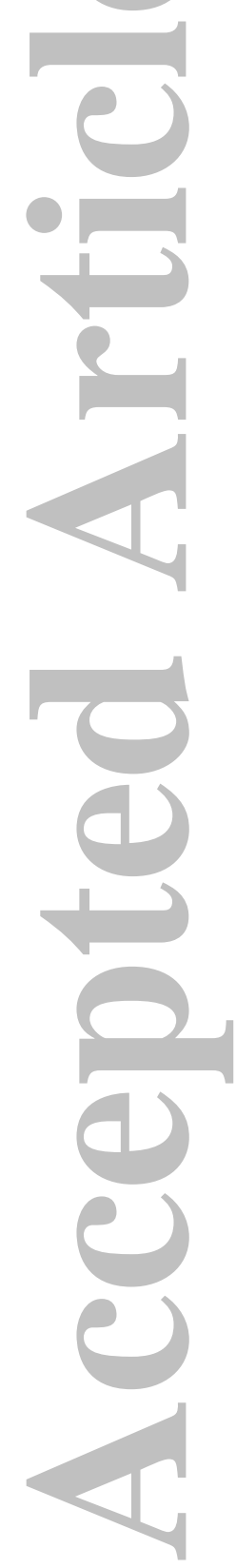


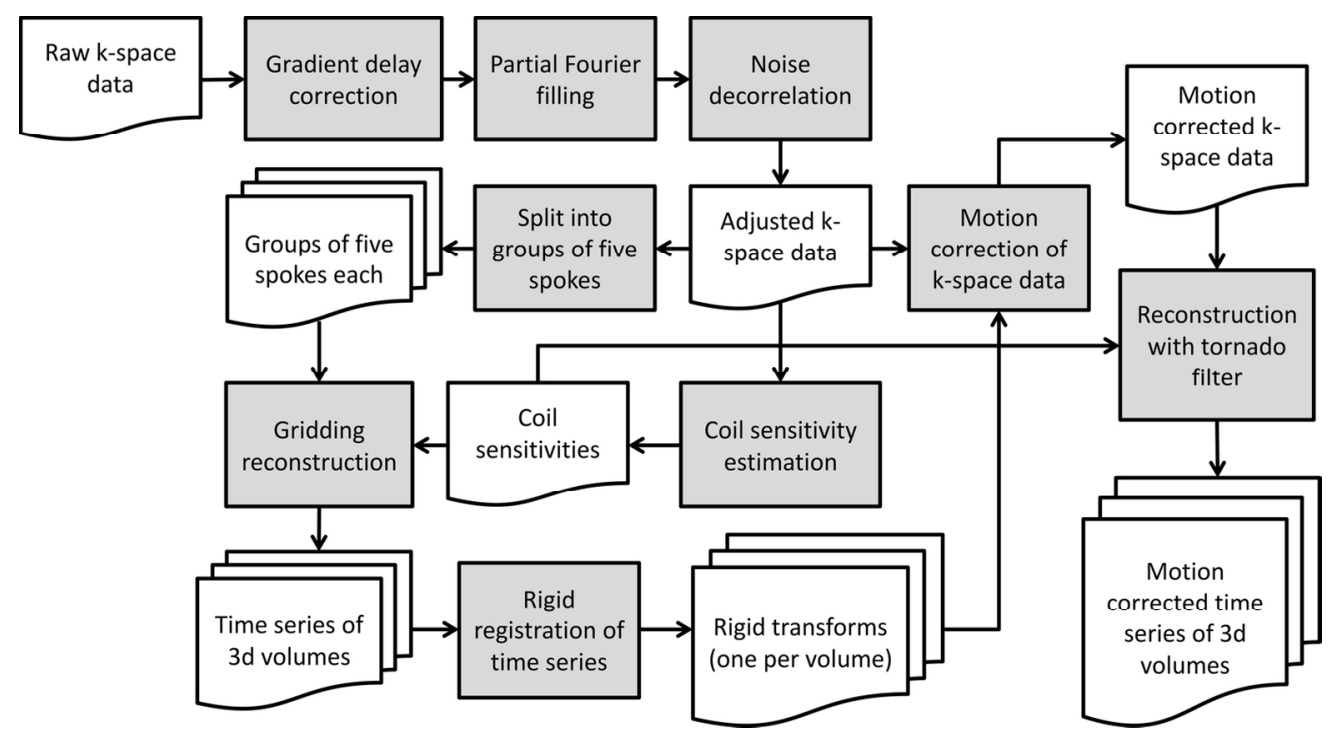

FIG. 1. Motion-corrected image reconstruction pipeline. The raw k-space data from the MRI scanner (upper left corner) is reconstructed into a motion-corrected DCE-MRI time series (lower right corner).

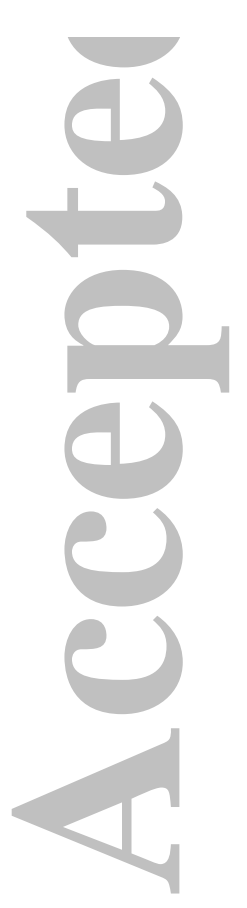

$69 \times 37 \mathrm{~mm}(600 \times 600 \mathrm{DPI})$ 


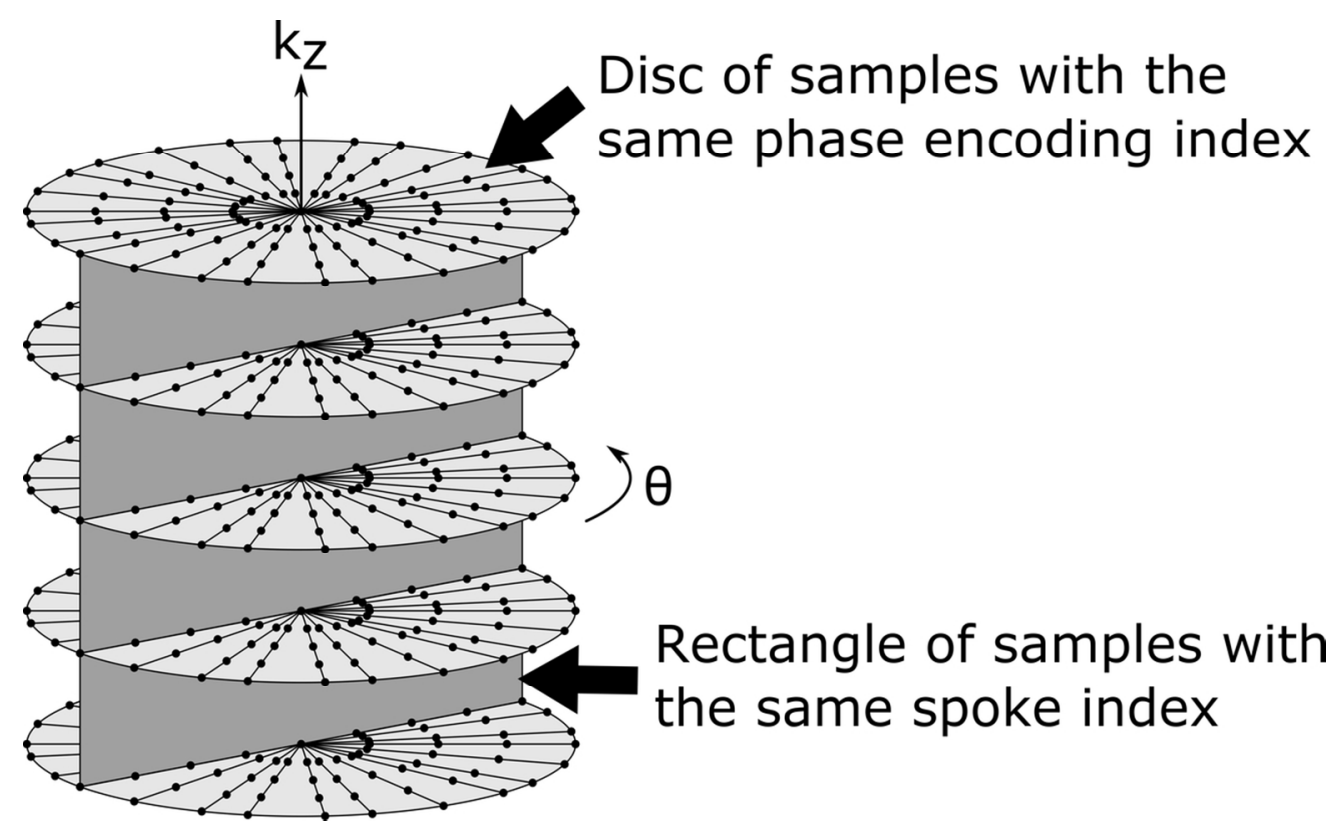

FIG. 2. Golden-angle stack-of-stars k-space sampling pattern used by the Radial VIBE sequence. Straight black radial lines represent readout lines in k-space. The top light gray disc touches all lines belonging to one particular k_z phase encoding index and the vertical dark gray rectangle touches all lines with a particular spoke index.

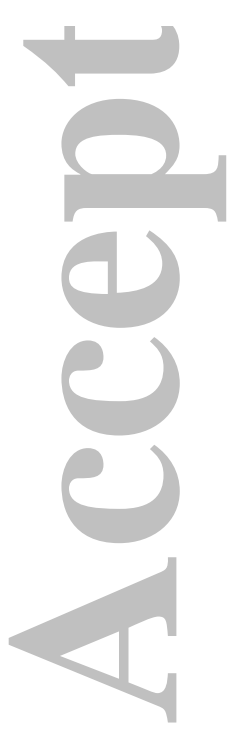

$54 \times 33 \mathrm{~mm}(600 \times 600 \mathrm{DPI})$ 

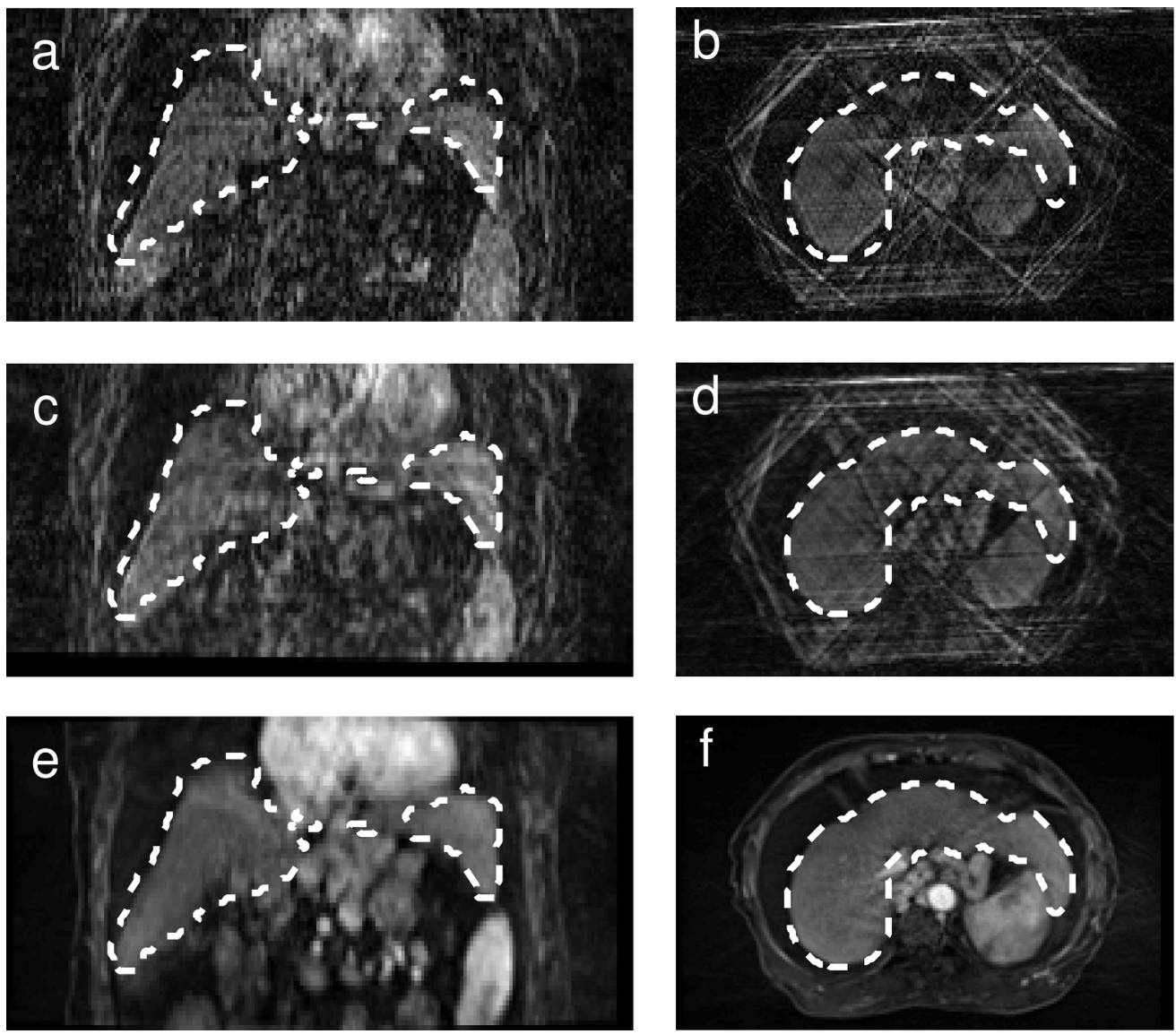

FIG. 3. Example of images reconstructed from five spokes before $(a, b)$ and after (c, d) registration. The reference volume on which the liver ROI used for registration was drawn $(e, f)$ is also shown. This reference image was reconstructed without motion correction from a large number of spokes. The dashed white line shows the position of the liver in the reference volume.

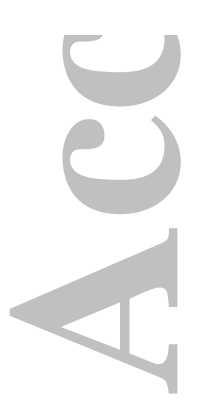

$109 \times 96 \mathrm{~mm}(600 \times 600 \mathrm{DPI})$ 


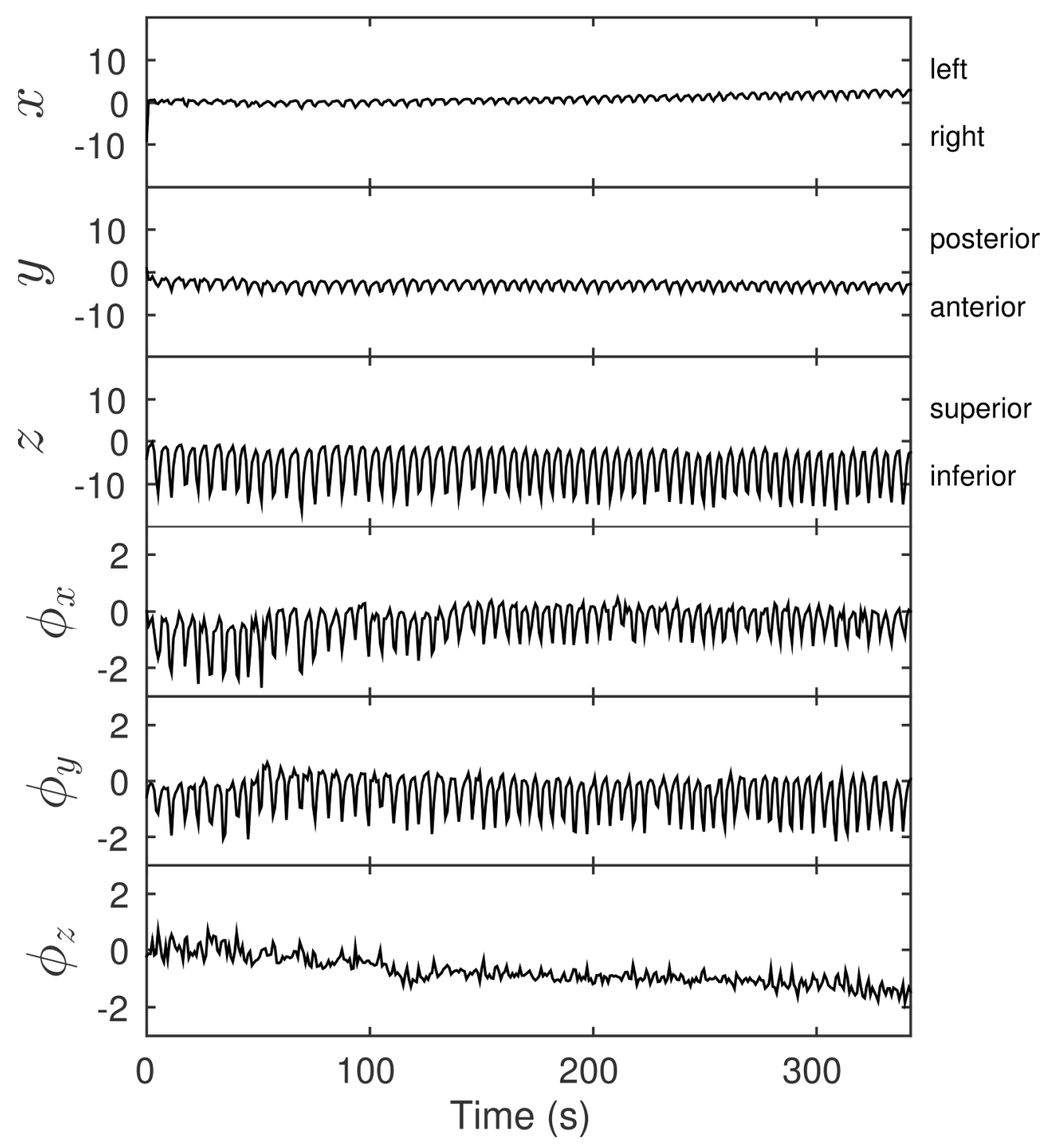

FIG. 4. Rigid-body motion signal components with respect to time. The first three components ( $x, y$ and $z-$ left, posterior and superior in $\mathrm{mm}$ ) describe the 3D translation of the center of the liver. The last three components ( $\phi_{-} \mathrm{x}, \phi_{-} \mathrm{y}$ and $\phi_{-} z$ in degrees) are the elements of an axis-angle representation of the rotation in degrees.

$112 \times 123 \mathrm{~mm}(600 \times 600 \mathrm{DPI})$ 

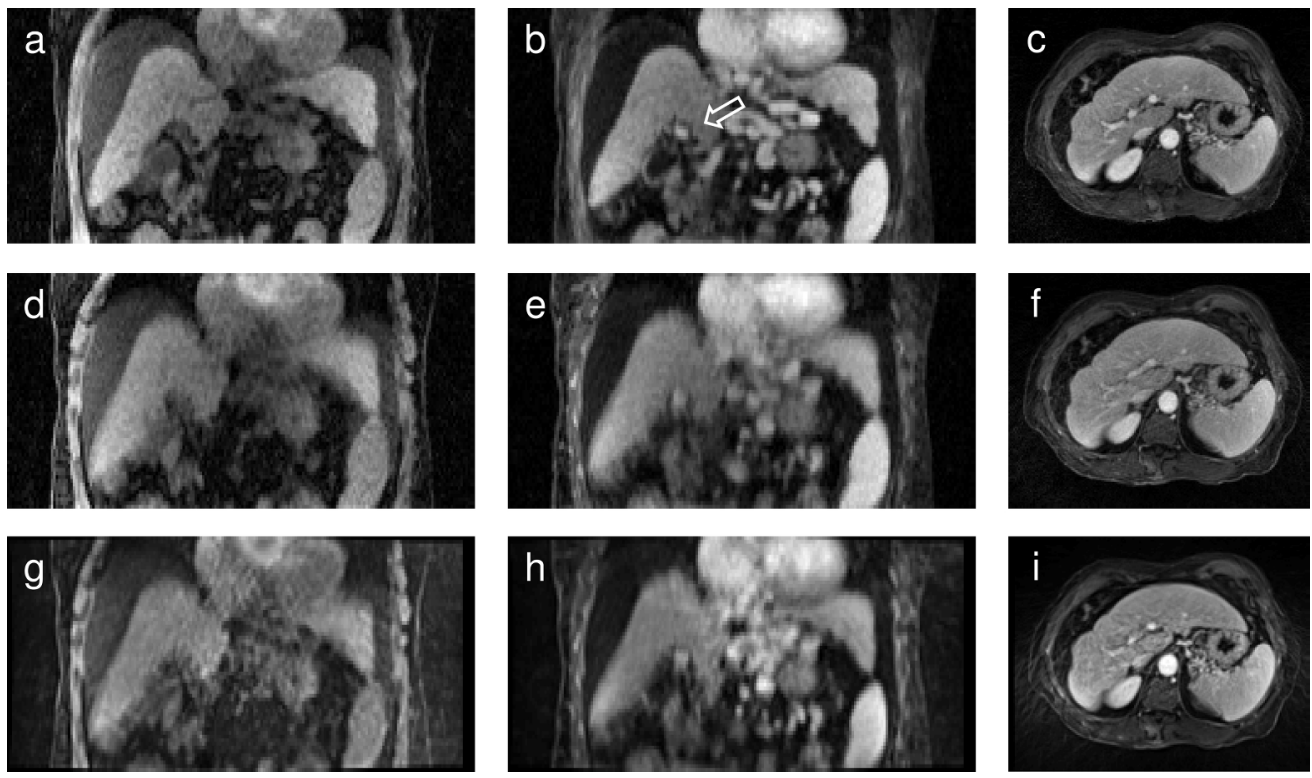

FIG. 5. Images reconstructed using the tornado filter with k-space motion correction ( $a, b, c)$ and without motion correction ( $d, e, f)$.Vendor-reconstructed images are shown in $(g)$ through (i). The left column (a, d, g) shows pre-contrast and the middle column $(b, e, h)$ post-contrast images in the coronal plane for one subject. The arrow in (b) points to the portal vein which appears as a bright spot. The right column (c, $\mathrm{f}, \mathrm{i}$ ) shows how motion artifacts appear in the transverse plane for another subject. Streak and motion artifacts

in (i) obscure the portal vein. In ( $f$ ) streak artifacts are reduced by the tornado filter and in (c) motion correction further enhances the contrast between the portal vein and surrounding tissue.

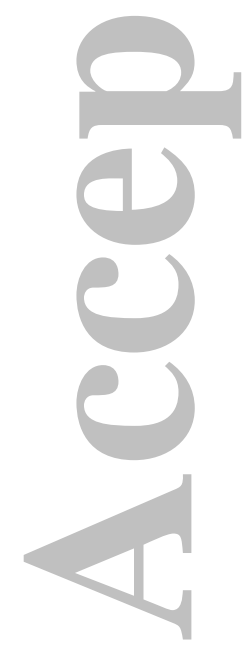

$110 \times 64 \mathrm{~mm}(600 \times 600 \mathrm{DPI})$ 

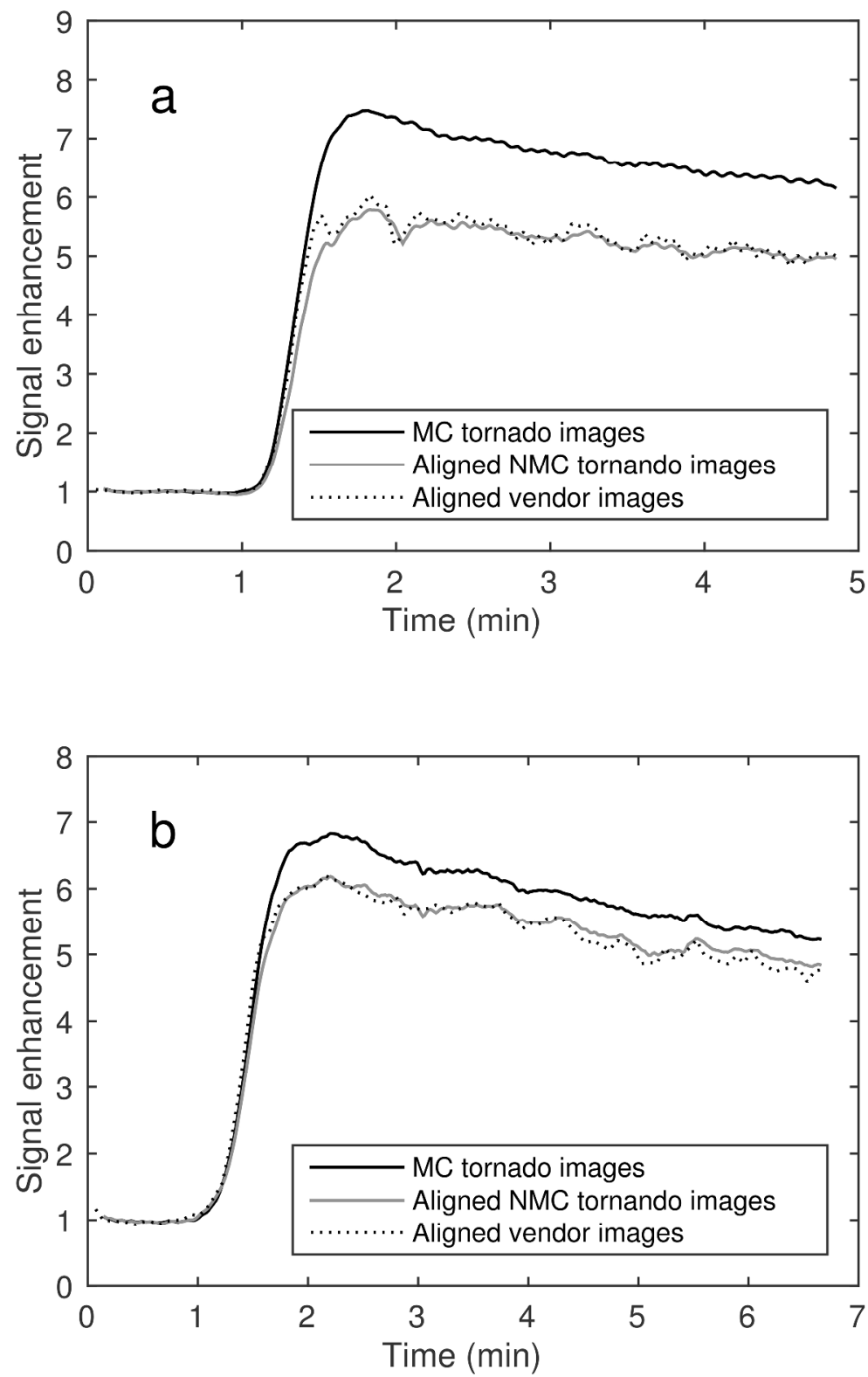

FIG. 6. Portal-venous input functions from two different patients are shown in (a) and (b). For each patient, input functions derived from motion-corrected (MC) and non-motion-corrected (NMC) images reconstructed with the tornado filter are shown as well as NMC images reconstructed by vendor software on the scanner. NMC images were aligned using rigid image registration before extraction of the input functions. Input function from MC images exhibit larger maximum signal enhancements and are smoother compared to their NMC counterparts. The temporal distance between images is about $1 \mathrm{~s}$ for the tornado images and about $3 \mathrm{~s}$ for the vendor images. However, the effective temporal resolution for the tornado images is about $7.7 \mathrm{~s}$ in the portal vein due to the width of the tornado filter.

$156 \times 249 \mathrm{~mm}(600 \times 600 \mathrm{DPI})$ 

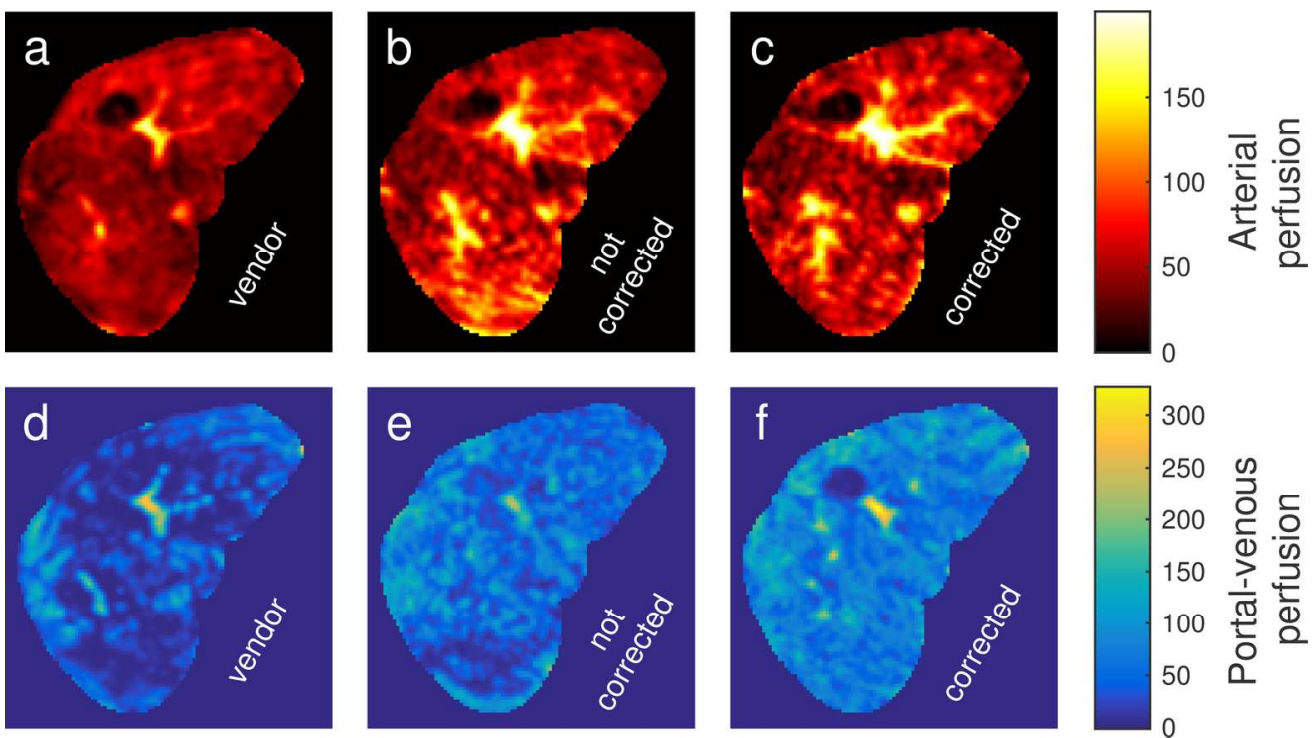

FIG. 7. Transverse arterial $(a-c)$ and portal-venous ( $d-f)$ perfusion maps calculated from DCE-MRI time series reconstructed by vendor software on the scanner without (first column), by a tornado filter without motion correction (second column) and by a tornado filter with motion correction (third column) for a subject. The portal-venous perfusion map in (e) exhibits severe artifacts caused by motion. Perfusion is given in $\mathrm{ml} /(100 \mathrm{ml} \cdot \mathrm{min})$.

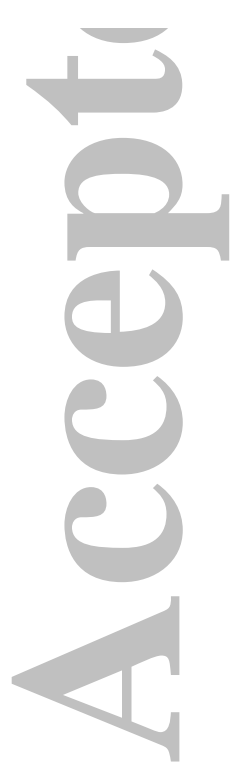

$84 \times 47 \mathrm{~mm}(600 \times 600$ DPI $)$ 

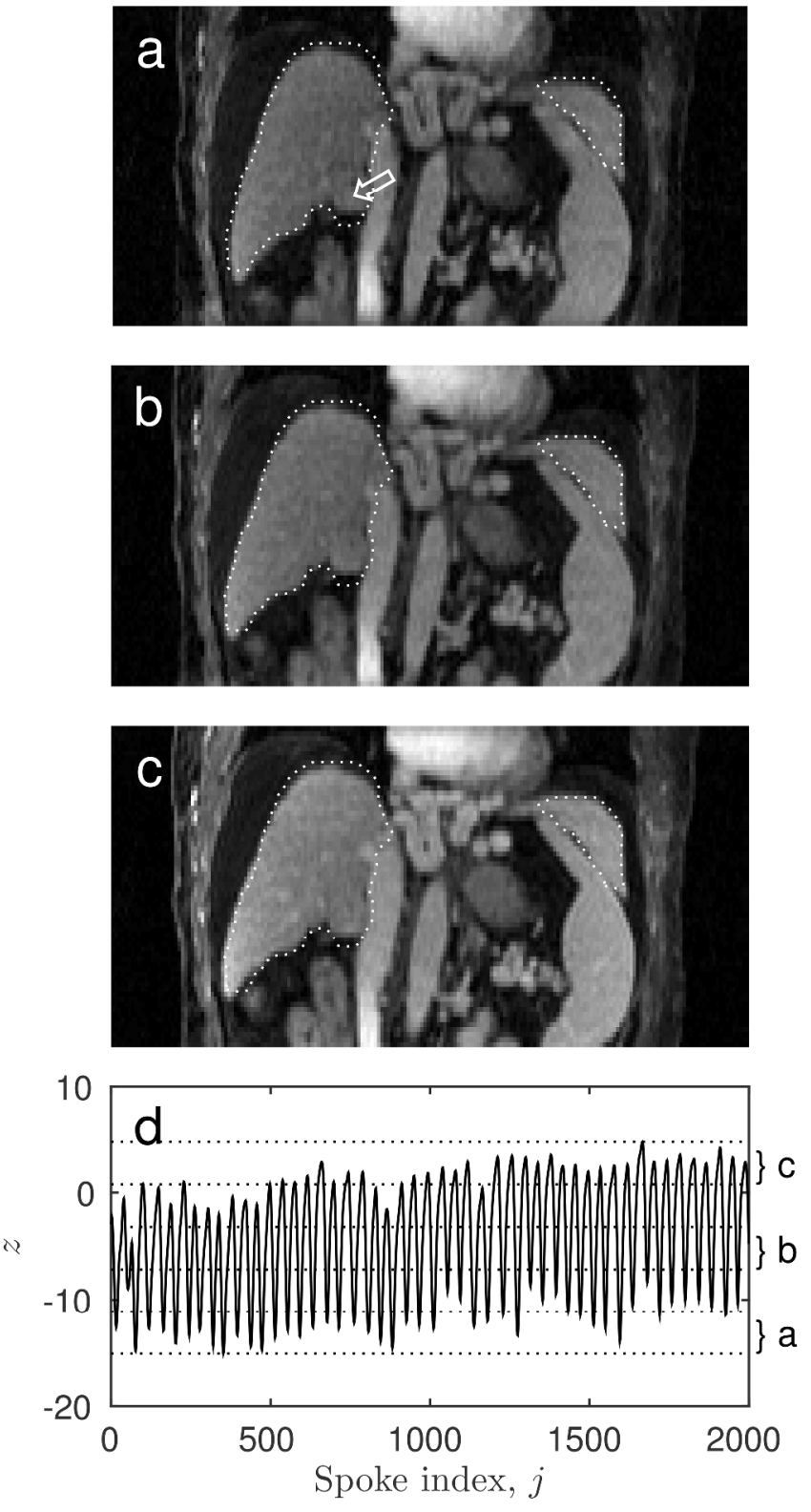

FIG. 8. Three ((a) first, (b) third and (c) fifth) of five reconstructed motion states from an example subject as well as the superior-inferior motion that was used for sorting k-space data into states (d). The dotted white ROI indicates the liver contour in the reference volume. The white arrow in (a) points to the location of a tumor. Because the motion states were corrected for rigid-body motion the remaining differences are the result of deformations, such as bending.

$158 \times 297 \mathrm{~mm}(600 \times 600 \mathrm{DPI})$ 